\title{
Uma Revisão sobre Estudos de Experiência Turística (2005-2016)
}

\author{
A review of Tourism Experience Studies (2005-2016) \\ Una Revisión sobre Estudios de Experiencia Turística (2005-2016) \\ Mariana de Freitas Coelho \\ Universidade Federal de Viçosa (UFV), Brasil \\ marifcoelho@gmail.com \\ Marlusa de Sevilha Gosling \\ Universidade Federal de Minas Gerais (UFMG), Brasil \\ mg.ufmg@gmail.com
}

DOI: https://doi.org/10.18472/cvt.19n2.2019.1485

Redalyc: http://www.redalyc.org/articulo.oa?

id $=115460585008$

Recepção: 05 Setembro 2017

Aprovação: 02 Maio 2019

\section{Resumo:}

Oferecer experiências em vez de serviços/produtos é um tópico que tem gerado atenção da academia e das organizações. As atividades turísticas possuem interseção com a experiência do visitante e o comportamento de consumo. Este trabalho tem o objetivo de revisar sistematicamente os estudos sobre a experiência turística. Para tanto, revisaram-se três periódicos de relevância nacional - Estudios y Perspectivas en Turismo (EyPT), Caderno Virtual de Turismo (CVT) e Revista Brasileira de Pesquisas em Turismo (RBTur) - bem como três periódicos de alto impacto internacional - Annals of Tourism Reserch (Annals), Tourism Management (TM) e Journal of Travel Research (JTR). As análises compreenderam três aspectos: i) métodos, ii) enfoque da unidade de análise e iii) abordagem da experiência dos artigos encontrados. Classificaram-se os estudos quanto à abordagem da experiência como: 1) vivência; 2) consumo; 3) transformações. Também são apontadas lacunas de pesquisa e orientações para pesquisas futuras.

Palavras-Chave: Turismo de experiência, Consumo, Vivência, Transformação.

\section{Abstract:}

Offering experiences rather than services/products is a topic that has generated attention from both the academia and the organizations. Tourism activities have a great intersection with visitor experience and consumer behavior. This paper aims to systematically review the studies on the tourism experience. Therefore, three national journals were reviewed - Estudios y Perspectivas en Turismo (EyPT), Caderno Virtual de Turismo (CVT) and Revista Brasileira de Pesquisas em Turismo (RBTur) as well as 3 journals of high international impact - Annals of Tourism Reserch (Annals), Tourism Management (TM) and Journal of Travel Research (JTR). The research analysis covered three aspects: i) methods, ii) approach to the unit of analysis and iii) the experience approach of the reviewed articles. We classified the studies regarding the approach of experience as: 1) life experience; 2) consumption; 3) transformations. Research gaps and guidelines for future research are also pointed out.

KEYWORDS: Tourism experience, Consumption, Life Experience, Transformation.

\section{ReSUMEN:}

Ofrecer experiencias en lugar de servicios/productos es un tema que ha generado la atención de la academia y de las organizaciones. Las actividades turísticas tienen una gran intersección con la experiencia del visitante y el comportamiento de consumo. Este trabajo tiene el objetivo de revisar sistemáticamente los estudios sobre la experiencia turística. Para ello, se revisaron 3 periódicos de relevancia nacional - Estudios y Perspectivas en Turismo (EyPT), Caderno Virtual de Turismo (CVT) y Revista Brasileira de Pesquisas en Turismo (RBTur) - así como 3 periódicos de alto impacto internacional - Annals of Turismo Reserch (Annals), Turismo Management (TM) y Journal of Travel Research (JTR). Las análisis comprendieron tres aspectos: i) métodos, ii) enfoque de la unidad de análisis y iii) abordaje de la experiencia de los artículos encontrados. Se clasificaron los estudios en cuanto al abordaje de la experiencia como: 1) vivencia; 2) consumo; 3) transformaciones. También se señalan lagunas de investigación y orientaciones para futuras investigaciones.

Palabras clave: Turismo de experiência, Consumo, Vivencia, Transformación. 


\section{INTRODUÇÃO}

A experiência é uma prática antiga, envolvendo rituais, cerimônias, teatro e até mesmo a arquitetura (BRUNNER-SPERDIN; PETERS, 2009). Desse modo, são muitos os elementos que se associam à experiência e um dos possíveis olhares sobre a mesma parte do entendimento da comercialização das experiências, pautadas na gestão e no marketing. A contribuição da perspectiva mercadológica da experiência envolve a identificação de estratégias e possibilidades de gerenciar a experiência do cliente por meio da customização e cocriação de atividades (PINE; GILMORE, 2011) e da geração de emoções e de eventos marcantes (SCHMITT, 2004) que promovam a transformação dos envolvidos.

A experiência é uma temática que tem sido abordada no Brasil e no exterior em diversas áreas de estudo, a saber: Administração, Contabilidade e Economia, e Ciências da Saúde (Psicologia) (MELO, 2012). Entre as poucas revisões nacionais efetuadas sobre o tema experiência, destacam-se dois estudos. Primeiramente, Melo (2012) fez um levantamento sobre o tema experiência em periódicos internacionais buscando as palavraschave Experience, Experiential, Experiential Value e Consumer Experience (Experiência, Experiencial, Valor experiencial e Experiência do Consumidor, respectivamente), separando as áreas de estudo entre (1) Administração, Contabilidade e Economia e (2) Ciências da Saúde. O autor identificou um aumento do interesse no tema, especialmente a partir dos anos 2000. Especificamente no contexto do comportamento do consumidor, houve um crescimento significativo de publicações internacionais a partir de 2007, isto é, a experiência e seus temas associados têm sido cada vez mais foco de pesquisas acadêmicas.

Melo (2012) também pesquisou a publicação sobre experiência em revistas nacionais, como a Revista de Administração Contemporânea (RAC), Revista de Administração de Empresas (RAE) e Revista Eletrônica de Administração (REAd) entre 2002 e 2011. Os resultados dos estudos indicam que publicações nacionais estão muito aquém das internacionais quanto ao número de publicações (MELO, 2012).

O segundo estudo é o de Ferreira e Teixeira (2013), confirmando a diferença entre publicações nacionais e internacionais ao ranquearem o número de citações da base Scopus de pesquisadores da experiência do consumidor de 36 países, em que o Brasil aparece em 22 lugar, com apenas 1,1\% das citações. Dos 320 artigos de experiência citados até 2012 na base Scopus, apenas quatro são de autores brasileiros, sendo os principais estudos publicados de autores dos Estados Unidos, Inglaterra, Taiwan e China (FERREIRA; TEIXEIRA, 2013). Em outras palavras, mesmo que exista um crescente interesse no tema Experiência no contexto das ciências sociais aplicadas no Brasil, os estudos de revisão de literatura citados apontam que a discussão do tema ainda está longe de esgotar-se.

Este estudo propõe um recorte particular dos estudos de experiência, isto é, a experiência turística. Ritchie e Hudson (2009) apontam três bases importantes sobre as experiências turísticas: 1) As experiências são originadas do contexto social e cultural dos indivíduos; 2) As experiências são multifacetadas; 3) As experiências são existenciais. Portanto, não há como falar de experiência turística sem enfatizar o turista. Mas, como aponta Panosso Netto (2007), o não turista também possui importância nesse domínio, justamente por mediar essa relação entre turista e destino, podendo, inclusive, gerar uma experiência autêntica. Autores da experiência turística também reforçam a necessidade de estudos de turismo de experiência em âmbito nacional, que ainda são escassos (PANOSSO NETTO; GAETA, 2010a; PEZZI; SANTOS, 2012; PEZZI; VIANNA, 2015).

A fim de se entender o estado da arte sobre o tema e encontrar lacunas nos estudos de experiência turística no contexto brasileiro e internacional, optou-se por pesquisar os estudos de experiência turística em seis periódicos acadêmicos selecionados por serem revistas com maior impacto ou mais bem avaliadas no contexto nacional. O estudo foi conduzido de janeiro a abril de 2017 e avaliou publicações de janeiro de 2005 a dezembro de 2016. Assim, o objetivo do artigo é avaliar os estudos de experiência turística nos principais periódicos de relevância nacional e internacional a partir de uma revisão de literatura do tema. Como objetivos secundários, tem-se o levantamento dos métodos dos artigos que abordam a experiência 
como processo de vivência (HENNES, 2002), e classificação das abordagens da experiência encontradas nesses artigos.

As contribuições do artigo são, sobretudo, teóricas visto que o estudo pode servir como referência e direcionamento para estudos na área. Além disso, a classificação das experiências em decorrência de processos de vivência, consumo ou transformação são algumas das reflexões propostas que vão além da teoria elencada e podem contribuir para direcionar pesquisas futuras.

\section{REVISÃO DE LITERATURA}

\subsection{DEFINIÇÕES DE EXPERIÊNCIA}

Uma experiência pode ser caracterizada como um fluxo constante de pensamentos (cognitivos) e sentimentos (emotivos) que ocorre durante os momentos de consciência em relação aos elementos da experiência (WALLS et al., 2011). A experiência também pode ser definida, em geral, sob duas dimensões distintas (HENNES, 2002; SCHMITT, 2000):

1. Experiência como conhecimento ou expertise

2. Experiência como um processo de vivenciar uma situação diferente, estranha, gerando emoções incômodas ou prazer.

O primeiro tipo de experiência se relaciona com experiências normais/automáticas da vida e é relativamente inconsciente por se tratar de um processo habitual, fluindo continuamente entre um momento e outro em um fluxo interrupto. Também se relaciona com o conhecimento que se adquire ao longo do tempo (SCHMITT, 2000), levando a uma expertise, ou ao "saber fazer" de uma determinada tarefa, atividade, ou algo que se relaciona com experiências comuns e automáticas que fluem continuamente entre um momento e outro sem interrupção. Uma pessoa pode ter experiência em atividades simples ou complexas, variando desde fazer café e dirigir à noite, a gerenciar equipes de vendas. Em outras palavras, a experiência nesse sentido se resume ao processo de saber fazer ou se conhecer algo em profundidade.

Já o segundo tipo de experiência surge quando o fluxo normal é interrompido por algo que chama atenção: um evento, uma mudança, algo fora do lugar, algo incomum, entre outros (HENNES, 2002). Pezzi e Santos (2012, p. 2) e Turner (1974) corroboram essa noção da experiência como sendo um agente transformador e formativo que "se inicia com choques de dor ou prazer vividos por um sujeito que, em seguida, busca dar sentido àquilo que percebeu [...]". É particularmente o segundo tipo de experiência que será destacado neste estudo, dado que a experiência turística envolve um contexto novo e de mudança. Porém, se levadas em consideração as ideias de Schmitt (2000), dor e prazer não são os únicos desdobramentos que uma experiência pode prover ao sujeito, mas um leque de sensações, sentimentos e formas de agir e de pensar.

De outra forma, Pezzi e Santos (2012) diferenciam os dois tipos de experiência por meio da passividade e aceitação dos acontecimentos, chamando a primeira de "mera" experiência e a segunda de "uma" experiência em que há maior participação do sujeito e "não tem início nem fim facultativo, desprendido da temporalidade cronológica" (PEZZI; VIANNA, 2015, p.168). Contudo, a literatura aponta que a experiência adquirida (experiência como conhecimento) pode remeter à participação ativa do participante, já que se associa à expertise do participante (BORGES, 2012; PALMER, 2010) e pode representar um aspecto ativo e produtivo de sua trajetória humana (MACIEL, 2010). Desse modo, não se trataria da passividade do sujeito, e, sim, da ruptura ou não com o estabelecimento de sentidos e ressignificação pessoal.

Há também uma diferenciação do conceito de experiência com base em dois vocábulos de origem alemã: Erlebinis e Erfahrung que se relacionam com a continuidade e ruptura da experiência (MACIEL, 2010; TRIGO, 2010). Conforme Maciel (2010), Erfahrung está associado à continuidade, onde o ser humano se encontra com as mesmas coisas para caracterizar sua história de vida. Para o autor, a experiência como 
Erfahrung pode ser representada por uma seta longilínea, havendo acúmulo de conhecimento por meio do contato direto com um objeto ao longo do tempo. De outra forma, Erlebnis é representado pelo autor como várias setas verticais, simbolizando rupturas e descontinuidade. Assim, Erlebnis seria a vivência de uma situação, ou evento que qualifica sua relação com o outro e consigo mesmo.

QUADRO 1

Definições de Experiência

\begin{tabular}{|c|c|c|}
\hline TIPOS & DIFERENCIACC̃O & AUTORES \\
\hline $\begin{array}{l}\text { Conhecimento Vivenciar } \\
\text { ou experimentar algo }\end{array}$ & $\begin{array}{l}\text { Fluxo continuo (Conhecimento) } \\
\text { ou fluxo interrompido } \\
\text { (Vivência) }\end{array}$ & $\begin{array}{l}\text { HENNES, 2002; } \\
\text { SCHMITT, 2000 }\end{array}$ \\
\hline $\begin{array}{l}\text { "mera" experiência "uma" } \\
\text { experiência }\end{array}$ & $\begin{array}{l}\text { Passividade e aceitação dos } \\
\text { acontecimentos. No segundo } \\
\text { tipo há maior participação do } \\
\text { sujeito. }\end{array}$ & PEZZI; SANTOS, 2012 \\
\hline \multirow[t]{2}{*}{ Erfahrung Erlebnis } & $\begin{array}{l}\text { Continuidade e ruptura Na } \\
\text { Erfahrung há vivência } \\
\text { continuada e na Erlebnis há } \\
\text { rupturas e descontinuidade }\end{array}$ & $\begin{array}{l}\text { MACIEL, 2010; } \\
\text { PANOSSO NETO; } \\
\text { GAETA, 2010b }\end{array}$ \\
\hline & $\begin{array}{l}\text { Erfahrung - Avaliação da } \\
\text { experiência Erlebnis - } \\
\text { experiência vivida }\end{array}$ & $\begin{array}{l}\text { CUTLER; } \\
\text { CARMICHAEL, } 2010\end{array}$ \\
\hline
\end{tabular}

Elaboração própria, 2017.

A conceituação de Maciel (2010) está de acordo com as propostas de Hennes (2002) e Schmitt (2000). Entretanto, Cutler e Carmichael (2010) definem que Erfahrung é que deve ser foco dos estudos de turismo, visto que é associado a uma avaliação da experiência; Erlebnis seria a experiência vivida de momento a momento. Nesse sentido, são necessárias pesquisas empíricas para entender melhor se ambas as dimensões da experiência Erfahrung e Erlebnis de fato se associam aos estudos de turismo. Por fim, há certo consenso na literatura de que existem pelo menos duas dimensões da experiência, representadas no Quadro 1.

A experiência é particular, mas acessível a todos dada sua subjetividade (TRIGO, 2010), por conseguinte, duas pessoas não podem vivenciar a mesma experiência (PINE; GILMORE, 1998), mas podem interferir na experiência de outros.

\section{MÉTODO}

Este estudo propõe uma revisão bibliográfica sistemática, com base nas diretrizes de Vasconcelos e Lezana (2014). Um primeiro filtro foi a escolha de periódicos para análise dos estudos. Com suporte do Arquivo Qualis Capes Quadriênio 2013-2016 ${ }^{[i]}$, três periódicos foram escolhidos para a identificação de estudos sobre experiência turística no contexto nacional. O recorte foi considerado necessário tendo em vista a pouca quantidade de revistas de turismo avaliadas pela Capes como B1 ou mais no Brasil até 2016.

1) Revista Estudios y Perspectivas en Turismo (EyPT), Qualis Capes A2 ${ }^{[i i]}$. Apesar de se tratar de uma revista argentina com publicações em espanhol, há muitas publicações de autores brasileiros. Pesquisaramse todas as edições de 2005 a 2016;

2) Revista Caderno Virtual de Turismo (CVT), Qualis Capes B1. Revista brasileira específica da área de turismo. Foi considerada porque já apresentava qualis B1 desde o triênio 2010-2012. Pesquisadas todas as edições de 2005 a 2016; 
3) Revista Brasileira de Pesquisa em Turismo (RBTur), Qualis Capes A2, escolhida por enfatizar pesquisas em âmbito nacional. A primeira publicação do periódico foi em 2008, sendo necessário considerar as publicações de 2008 a 2016.

Além disso, foi conduzida uma revisão nos mesmos parâmetros em abril de 2017 em três principais periódicos internacionais de turismo. Apesar da EyPT não ser um periódico nacional, neste artigo, as revistas 1,2 e 3 serão nomeadas como periódicos de relevância nacional. Por outro lado, as revistas 4,5 e 6 contribuirão para o entendimento do cenário internacional. O critério de seleção dos periódicos internacionais abrangeu os periódicos com fator de impacto mais alto da área de Turismo, Hospitalidade e Gestão do Scimago.

4) Annals of Tourism Research (Annals), é um periódico britânico de ciências sociais que enfatiza as perspectivas acadêmicas do turismo, extrato Q1, fator de impacto $2,658^{[i i i]}$;

5) Tourism Management (TM), periódico britânico, referência nos temas de planejamento e gestão de viagens e turismo, extrato Q1, fator de impacto $2,450^{[\mathrm{iv}]}$.

6) Journal of Travel Research (JTR), periódico líder entre os artigos na subárea de Turismo, Hospitalidade e Gestão do Scimago Journal and Country Rank. Também britânico, extrato Q1 e fator de impacto $3,490^{[\mathrm{v}]}$.

O segundo filtro foi a escolha de palavras-chave, que consistiram em: experiência, experiências, experience, experiencial e experiência de consumo. Após a busca dos artigos, cada artigo foi separado por revista e salvo no software Mendeley para análise de seu conteúdo. As palavras-chave foram pesquisadas por periódico, tanto na base de pesquisa da própria revista por título e resumo, quanto em cada volume específico para averiguação do termo no título do artigo. Essas buscas utilizando os dois filtros (periódicos e palavras-chave) geram o portfólio bibliográfico deste estudo.

Em seguida, elencou-se o número de publicações encontradas por meio do levantamento de artigos com os termos pesquisados em seu título. O processo envolveu o acesso integral a cada periódico e busca dos artigos almejados por volume da revista. Novas pesquisas por títulos buscaram evitar erros e confirmar o levantamento numérico dos artigos.

Em um segundo momento, prosseguiu-se com a análise dos seguintes itens: i) métodos, ii) enfoque da unidade de análise e iii) abordagem da experiência dos artigos encontrados. Quanto aos métodos, os artigos foram separados em artigos teóricos, qualitativos, quantitativos ou mistos. $\mathrm{O}$ enfoque da unidade de análise envolveu aspectos geográficos, e a diferenciação quanto à abordagem de atrativo, destino, regional, país, crosscountry, temático, projeto ou negócio. Por fim, os artigos foram classificados em relação à abordagem da experiência, isto é, se a experiência turística foi tratada como 1) um processo de vivência, 2) um processo de consumo, ou 3) um processo de transformação.

Contudo, percebeu-se que alguns artigos desvirtuavam da área de interesse, ou seja, não se tratavam de artigos que abarcam a experiência como processo de vivência. Por isso, foi necessário excluir alguns artigos a fim de agrupar os estudos para encontrar as principais lacunas de estudos nacionais e internacionais. Após uma breve avaliação do título, resumo e leitura flutuante de cada artigo, notou-se que parte deles trabalha a experiência como caso, exemplo ou prática, por exemplo: "Turismo comunitário e internet: análise dos sites das experiências do Brasil” e “¿De qué depende el éxito de las investigaciones en turismo? La experiencia de especialistas brasileños”. Logo, seis artigos foram descartados para análises posteriores ${ }^{\text {[vi] }}$ e apenas artigos que enfatizavam a experiência de consumo e a experiência turística foram avaliados quanto ao método empregado.

O mesmo aconteceu no contexto internacional, ainda que com um número muito inferior de artigos, quando considerado o todo. No periódico Annals, dois artigos foram retirados das análises seguintes: 1) Okumus, Levent e Roper (2007), o qual se refere a um estudo que trabalha a experiência como conhecimento a partir da prática e 2) Garay e Cànoves (2011), trataram a experiência como um caso no artigo. Já no periódico Tourism Management, quatro artigos foram retirados de análises posteriores: Noveli, Schmitz e Spencer (2006) e Özturk (2009), por se tratarem de artigos que abordam a experiência como caso, e Pansiri (2009) e Tseng (2012) por abrangerem a experiência como conhecimento pessoal advindo da prática. Assim, 
foram analisados apenas os artigos que tinham como pano de fundo a experiência turística e/ou a experiência de consumo.

\section{RESULTADO DO NÚMERO DE PUBLICAÇÕES}

Com o intuito de verificar possíveis diferenças entre as publicações de experiência turística, adotou-se uma análise comparativa entre os periódicos de relevância nacional versus internacional.

TABELA 1

Número de Publicações de Experiência Turística em periódicos de relevância nacional

\begin{tabular}{|c|c|c|c|c|c|c|c|c|c|c|c|c|}
\hline \multicolumn{13}{|c|}{ Anos das publicações } \\
\hline 200 & 2006 & 2007 & 2008 & 2009 & 2010 & 2011 & 2012 & 2013 & 2014 & 2015 & 2016 & TOTAL \\
\hline EyPT & & & & & 3 & 1 & 2 & 1 & 1 & & 2 & 10 \\
\hline CVT & & & 1 & 1 & & & 1 & & 2 & 2 & 1 & 8 \\
\hline RBTur NA & NA & NA & & 2 & & & 1 & 1 & 1 & 1 & 2 & 8 \\
\hline Total & & & 1 & 3 & 3 & 1 & -4 & 2 & 5 & 3 & 5 & 26 \\
\hline
\end{tabular}

\footnotetext{
Elaboração própria

Nota NA = não se aplica; EyPT $=$ Estudios y Perspectivas em Turismo; CVT =

Caderno Virtual de Turismo; RBtur = Revista Brasileira de Pesquisa em Turismo.
}

Ao se averiguar as edições dos últimos 11 anos da EyPT, foram encontrados 10 artigos publicados com a palavra experiência(s) em seu título. Durante o mesmo período, oito artigos foram publicados na CVT. A RBTur, que iniciou seus trabalhos em 2008, publicou oito artigos relacionados à experiência turística até o último volume do ano de 2016. Com isso, identificou-se um total de 26 artigos acadêmicos nos periódicos selecionados, os quais confirmam a evolução de publicações com o tema pesquisado a partir de 2008 (Tabela 1).

Por um lado, a Tabela 1 confirma a tendência apontada por Ferreira e Teixeira (2013) de aumento das publicações relativas à experiência de consumo nos últimos anos. Por outro, corrobora a necessidade de mais estudos sobre o tema no Brasil, por não demonstrar crescimento significativo no número de publicações correlatas.

De forma complementar, encontrou-se um número superior de publicações sobre experiência turística no contexto internacional, conforme a Tabela 2. Entre 2005 e 2016, foram identificados 38 artigos com o termo experiência e/ou derivados no título da Annals of Tourism Research e 39 no Journal of Travel Research. De outro modo, o número de estudos com as mesmas características no periódico Tourism Management revelou-se muito mais expressivo, totalizando 69 publicações. Assim, ao todo, os três periódicos somaram 146 artigos sobre experiência no período de 2005-2016.

TABELA 2

Número de Publicações de Experiência Turística em periódicos de relevância internacional

\begin{tabular}{|c|c|c|c|c|c|c|c|c|c|c|c|c|c|}
\hline \multicolumn{14}{|c|}{ Anos das Publicações } \\
\hline Periódico & 2005 & 2006 & 2007 & 2008 & 2009 & 2010 & 2011 & 2012 & 2013 & 2014 & 2015 & 2016 & Total \\
\hline Annals & 2 & 1 & & 1 & 4 & 3 & 5 & 6 & 4 & 4 & 5 & 3 & 38 \\
\hline TM & 5 & 4 & 1 & 2 & 3 & & 8 & 11 & 8 & 12 & 6 & 9 & 69 \\
\hline JTR & 2 & 2 & 3 & & 2 & 6 & 1 & 4 & 7 & 2 & 5 & 5 & 39 \\
\hline Total & 9 & 7 & 4 & 3 & 9 & 9 & 14 & 21 & 19 & 18 & 16 & 17 & 146 \\
\hline
\end{tabular}


Assim, ao visualizar a Figura 1, percebe-se que o número de publicações sobre experiência turística é superior em periódicos internacionais. Isso demonstra que o tema experiência turística é mais pesquisado no exterior do que nacionalmente. Porém, deve-se ter em mente que o número de volumes das revistas internacionais também é superior. Além disso, a figura também demonstra que, em geral, a partir de 2009, há de fato uma ascensão do tema nacional e internacionalmente, com um pico em 2012. Entretanto, se uma avaliação anual for considerada, não há um aumento regular das publicações sobre experiência, ou seja, não se pode dizer que a experiência turística vem ganhando mais atenção e um maior número de publicações acadêmicas a cada ano. (Error 1: La referencia: Figura 1 está ligada a un elemento que ya no existe)

Após a análise inicial, buscou-se entender o conteúdo dos artigos que citavam a experiência e termos correlatos em seu título. Com isso, prosseguiu-se com a análise dos métodos, enfoque e abordagem.

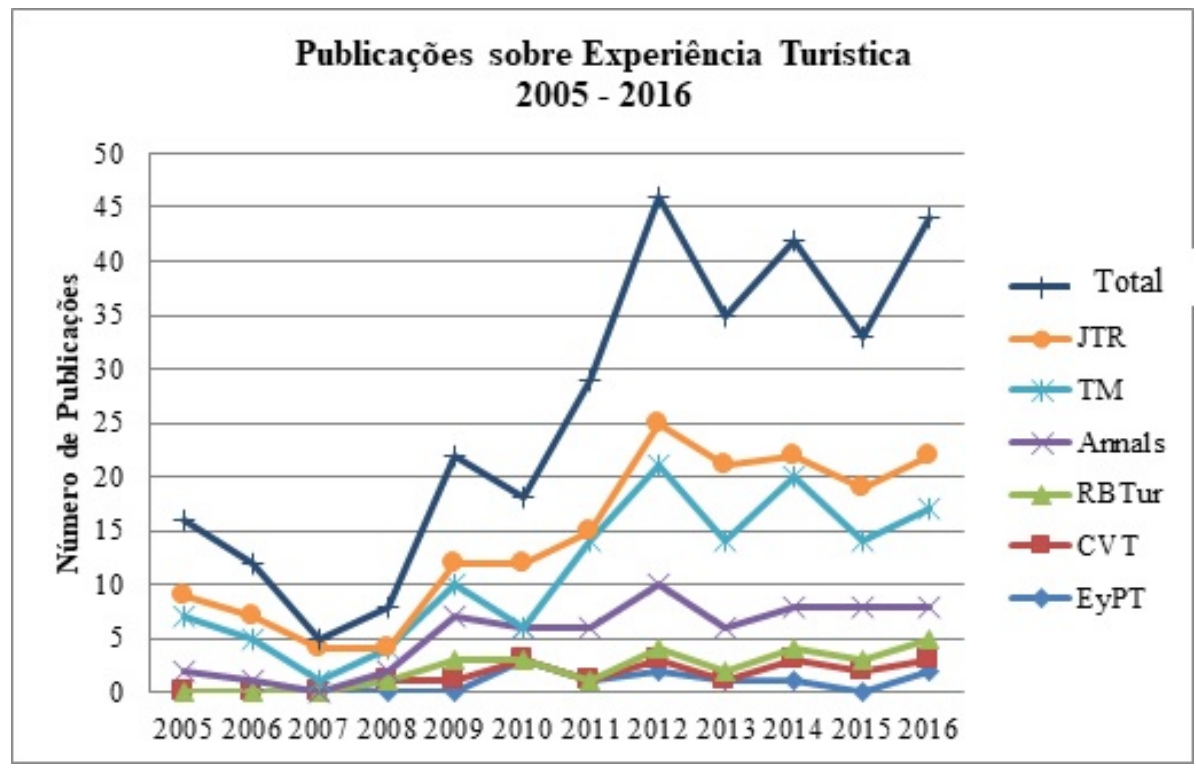

FIGURA 1

Publicações de Experiência Turística 2005-2016

Elaboração própria, 2017.

\subsection{MÉTODOS UTILIZADOS NOS ESTUDOS DE EXPERIÊNCIA TURÍSTICA}

Quanto aos métodos, em geral, os trabalhos pesquisados possuem natureza empírica, com pesquisa de campo e cunho exploratório (Tabela 3). Apenas $8,18 \%$ das publicações revisadas apresentaram natureza teórica, ao passo que $91,82 \%$ dos artigos avaliados apresentam uma pesquisa aplicada.

No contexto brasileiro, tem-se que muitos estudos estão buscando explorar o tema a partir de análises qualitativas. Há também pesquisas de caráter exclusivamente teórico, buscando discutir a experiência em algum contexto específico como a qualidade da experiência (KRAUSE, 2014) e experiência de lazer e ócio (CHEIBUB; MELO, 2010). Uma das barreiras encontradas foi a falta da descrição específica da análise dos dados (por exemplo, se análise de conteúdo, análise do discurso, análise semiótica, entre outras), dificultando a classificação do método de pesquisa.

Nos estudos internacionais, o número de estudos qualitativos e quantitativos é mais equilibrado, com predomínio um pouco superior de estudos quantitativos (59) em comparação com qualitativos (54). Os achados sobre os métodos dos estudos apontam para a natureza complexa e multifacetada do tema experiência, de forma que todas as abordagens são pertinentes para se entender o fenômeno. Assim, temse também uma lacuna que envolve o uso de pesquisas mistas para pesquisadores da área, visto que, em geral, apenas $13,21 \%$ dos estudos apresentaram métodos mistos. Ainda menor é o número de artigos teóricos 
publicados. É necessário ressaltar que apenas três estudos entre os 159 estudados são de revisão bibliográfica, todos publicados com um mesmo coautor no JTR: Durko e Petrick (2013), Stone e Petrick (2013a, 2013b).

TABELA 3

Método de pesquisa dos artigos de Experiência Turística

\begin{tabular}{lllllll}
\hline Periódico/ Método & Quanti & Quali & Misto & Teórico & Total & $(\%)$ \\
\hline CVT & 1 & 3 & 2 & 1 & 7 & 4,40 \\
EyPT & - & 5 & 1 & 1 & 7 & 4,40 \\
RBTur & 1 & 2 & - & 2 & 5 & 3,14 \\
Annals & 10 & 19 & 3 & 4 & 36 & 22,64 \\
TM & 27 & 27 & 10 & 1 & 65 & 40,88 \\
JTR & 22 & 8 & 5 & 4 & 39 & 24,53 \\
Total & 61 & 64 & 21 & 13 & 159 & \\
(9) & 38,36 & 40,25 & 13,21 & 8,18 & & \\
\hline
\end{tabular}

Elaboração própria, 2017.

Nos estudos quantitativos ou mistos das revistas nacionais, a análise descritiva dos dados busca identificar o perfil das unidades de análise, sobretudo os turistas. Assim, as análises quantitativas nos periódicos nacionais, em geral, expõem gráficos e contemplam amostras não probabilísticas. Com isso, tanto a utilização de pesquisas mistas quanto a variação dos métodos de pesquisas, sobretudo quantitativamente, podem agregar conhecimento ao tema. Trabalhos recentes como o de Carvalho et al. (2016) - pré-experimento e Gosling, Silva e Coelho (2016) - Modelagem de Equações Estruturais (SEM) são exemplos de avanços. Logo, uma lacuna encontrada é a possibilidade de variação na aplicação de métodos para estudos de experiência turística no Brasil.

Ademais, especificamente sobre os estudos publicados em periódicos estrangeiros, percebe-se uma preferência nítida nas abordagens utilizadas. Em estudos qualitativos, é recorrente a abordagem por meio de etnografias (ANDRIOTIS, 2010; BROWN, 2009; CARLISLE et al., 2013; CHRONIS, 2015; ES; REIJNDERS, 2016; KOMPPULA; ILVES; AIREY, 2016; PALMER, 2005) e seus desdobramentos mais recentes, como autoenografia (KOMPPULA; GARTNER, 2013; MACKENZIE; KERR, 2013) e netnografia (JANTA et al., 2011; TUSSYADIAH; FESENMAIER, 2009). Também há diversos autores que utilizam as entrevistas como método de coleta de dados (KIDRON, 2013; PAN, 2012; RITCHIE; TUNG; RITCHIE, 2011; SHANI; URIELY, 2012; TAN; KUNG; LUH, 2013), bem como grupos de foco (BUZINDE et al., 2014; COGHLAN; FILO, 2013; SHARPLEY; JEPSON, 2011). Há também estudiosos que usam o estudo de caso (BUCHMANN; MOORE, 2010; XUE; KERSTETTER; BUZINDE, 2015) e apenas dois estudos utilizaram técnicas de experimento (CROUCH et al., 2007; SØRENSEN; JENSEN, 2015).

Os métodos quantitativos de publicações internacionais apresentam o survey como método predominante de coleta de dados. A análise dos dados faz uso, principalmente, da Modelagem de Equações Estruturais, análise fatorial e estatística descritiva. Enquanto isso, os métodos mistos normalmente apresentam estudos qualitativos em um primeiro momento, com objetivo de contribuir para a construção do questionário, seguidos de survey.

\subsection{ENFOQUE DA UNIDADE DE ANÁLISE NOS ESTUDOS DE EXPERIÊNCIA TURÍSTICA}

O enfoque das unidades de análise em periódicos de relevância nacional se deu em destinos ou regióes turísticas e projetos sociais, demandando estudos de segmentos específicos e tipologias turísticas (turismo de natureza, ecoturismo, turismo cultural e outros). Quatro trabalhos pesquisaram destinos internacionais 
(CARVALHO et al., 2016; GÂNDARA; BREA; MANOSSO, 2013; HORODISKI; CRISITNA; GÂNDARA, 2012; SAMPAIO et al. 2014), havendo espaço para trabalhos de nível nacional e estudos comparativos entre países como o proposto por Sampaio et al. (2014).

Três pontos chamam atenção ao avaliar o enfoque das unidades de análise nos periódicos internacionais. Primeiramente, há uma nítida concentração dos estudos na América do Norte (Estados Unidos), Ásia (China, Taiwan e Coreia), Oceania (Austrália e Nova Zelândia), e em países europeus (Inglaterra, Espanha e Portugal) (Figura 2).

Ainda sobre a concentração geográfica, poucas publicações dos periódicos Annals e do Tourism Management abrangeram países da América do Sul (CUTLER; CARMICHAEL; DOHERTY, 2014; MACKENZIE; KERR, 2013), América Central (FERDINAND; WILLIAMS, 2013) e África (CARLISLE et al., 2013). No caso do Journal of Travel Research, nenhum artigo contemplou a América Latina ou África.

Esse resultado é interessante porque demonstra a falta de inserção de pesquisas sobre destinos em desenvolvimento, como Brasil, Rússia, Índia, África do Sul e até de destinos economicamente consolidados, como Canadá e Alemanha. Entre as possíveis justificativas da falta de publicação em regiões específicas estão as barreiras de idioma, a falta de apoio de instituições de pesquisa e a necessidade de associação/parcerias entre pesquisadores de locais distintos. Sobre o último argumento, dos 140 artigos avaliados nos periódicos internacionais, 10 apresentaram estudos em mais de um país, isto é, estudos cross-country (CARLISLE et al., 2013; CHARTERS; FOUNTAIN; FISH, 2009; CORREIA; KOZAK, 2016; ES; REIJNDERS, 2016; HERSH, 2016; HOSANY et al., 2015; KIM; RITCHIE, 2014; KOMPPULA; GARTNER, 2013; PORIA, 2006; PREBENSEN; KIM; UYSAL, 2016).

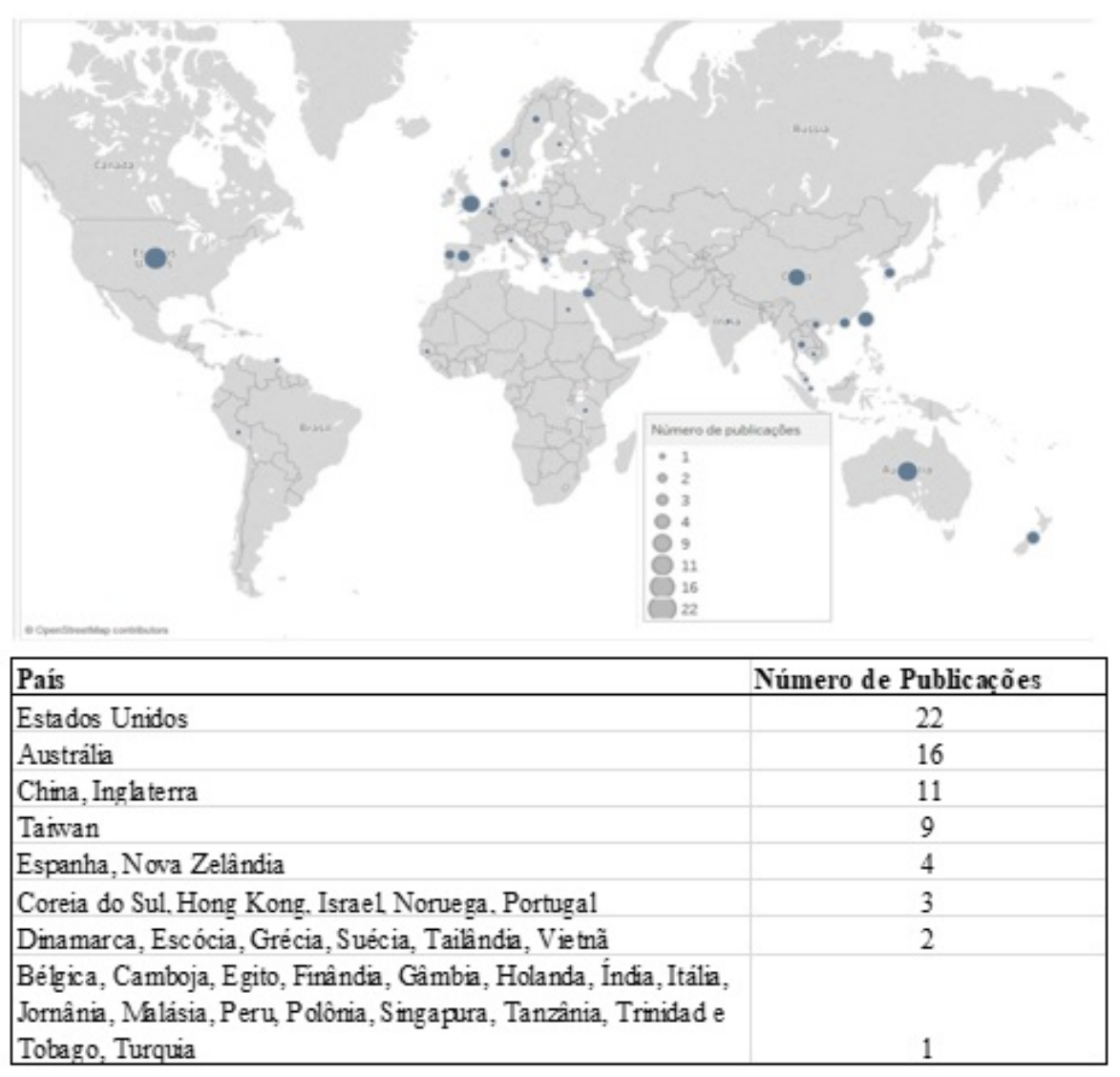

FIGURA 2

Mapa de Publicações Internacionais sobre Experiência Turística Elaboração própria, 2017. Elaborado por meio do software Tableau. 
Em segundo lugar, no contexto internacional, percebe-se uma tendência de maior número de estudos temáticos, principalmente os que envolvem tipos particulares de motivações turísticas: Turismo Sombrio (Dark Tourism), Turismo de Animais Selvagens (Wildlife Tourism), Turismo de Peregrinação (Pilgrimage Tourism), Turismo Social (Social Tourism), Turismo Voluntário (Volunteer Tourism) e Turismo Sexual (Sex Tourism). Há também ênfase nas atividades relacionadas ao turismo como: uso de tecnologia e dança, bem como participação em festivais e parques temáticos (Theme Parks). Além disso, grupos de consumidores específicos, como pessoas com deficiência (DANIELS; RODGERS; WIGGINS, 2005; SMALL; DARCY; PACKER, 2012), intercambistas (BEHNKE; SEO; MILLER, 2014) e grupos de viajantes que conheceram destinos internacionais foram alvos de alguns estudos (BOJANIC, 2011; CHANG; KIVELA; MAK, 2011; PRENTICE et al., 1998).

A terceira particularidade é o fato de que artigos com enfoque nos Estados Unidos tendem a ser menos direcionados, pesquisando a percepção de consumidores de uma forma mais ampla (BERDYCHEVSKY; GIBSON, 2015; CHEN; HUANG; PETRICK, 2016; MATHIS et al., 2016; SCARINCI; PEARCE, 2012) do que estudos chineses e australianos, por exemplo.

\subsection{ABORDAGEM DA EXPERIÊNCIA TURÍSTICA}

Uma das análises do artigo compreende a abordagem da experiência. Haja vista que a experiência é multidimensional, há diversas formas de abordá-las durante as pesquisas.

De acordo com Hennes (2002) e Schmitt (2000), a experiência é um processo de vivência. Em outras palavras, é necessário experimentar, sentir e participar de uma ou várias atividades para experimentar algo. Em geral, essa é a abordagem mais comum para diversos estudos de experiência turística. Estudos que abordam a experiência como vivência possuem cunho abrangente, principalmente aqueles que se referem a um destino turístico ou região, isto é, parte-se da premissa de que só existe experiência se uma avaliação geral de tudo aquilo que foi vivido durante a viagem for ponderado. Uma particularidade da abordagem da experiência como vivência foi a identificação de estudos que especificam a experiência como algo memorável, isto é, uma vivência capaz de ser levada na memória (ALI; RYU; HUSSAIN, 2016; KIM; JANG, 2016; KIM; RITCHIE, 2014; LEE, 2015; PARK, 2011; TUNG et al., 2016; TUNG; RITCHIE, 2011).

De outra forma, a experiência também é estudada com o pano de fundo de consumo. Seja na perspectiva dos prestadores de serviço, seja dos consumidores, a experiência como consumo se aplica em situações em que a compra/consumo é determinante, e, por vezes, central para a geração do estranhamento/ ruptura/diferenciação apontados por autores que especificam a experiência como Erlebnis (MACIEL, 2010; PANOSSO NETTO; GAETA, 2010b).

De maneira mais recente e tímida, a experiência tem sido descrita como um processo transformador. Nesse caso, a experiência propicia a ruptura e a transformação, isto é, alterações em algum nível no aprendizado, comportamento, estilo de vida, padrões de consumo, qualidade de vida e bem-estar. Mas também pode haver transformações em um contexto mais amplo, como socialização (TRIGO, 2010) e mudanças sociais.

O framework da Figura 3 contribui para a visualização de que há interseções entre as abordagens da experiência. Os números representam o número de artigos que foram classificados em cada um dos tipos de abordagem da experiência turística. 


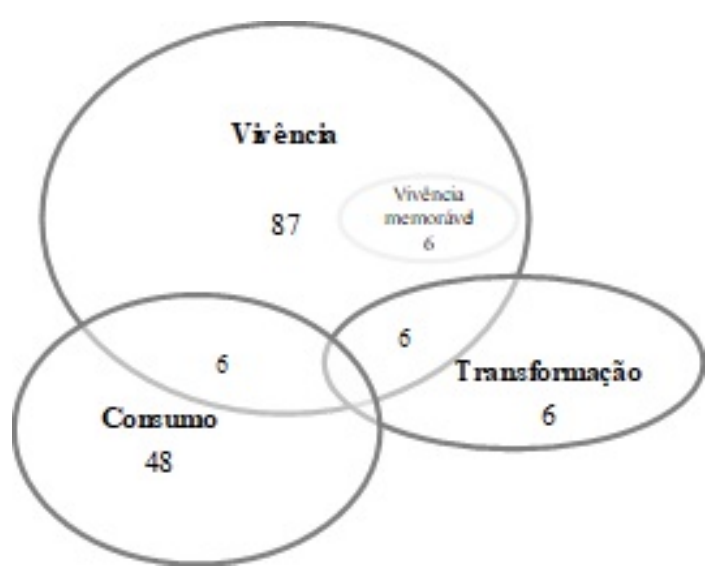

FIGURA 3

Tipos de abordagem da Experiência em estudos de Experiência Turística Elaboração própria, 2017.

Assim, este estudo demonstra a importância de que os autores se posicionem sobre o tipo de abordagem de experiência que adotam em seus trabalhos. Isso facilitaria o entendimento e evitaria a má interpretação do termo "experiência" na academia, evitando o uso deste para se referir a casos e exemplos práticos. Ademais, demonstra-se que a interseção da experiência com estudos de comportamento do consumidor e gestão possui diversos caminhos e diferenciações a serem adotados pelos autores.

\section{LACUNAS, PESQUISAS FUTURAS E LIMITAÇÕES}

Finalmente, avaliou-se que o estudo das experiências turísticas demanda detalhamento em diversas áreas do conhecimento, existindo oportunidade de pesquisas em:

a) estudar a perspectiva do nível de negócio, sobretudo com foco nos empreendedores e gestores de organizações de destinos turísticos;

b) conhecer as particularidades de setores determinados (companhias aéreas, agências de viagem, museus, artesãos, entre outros) e tipologias turísticas (turismo de natureza, turismo de aventura, turismo cultural, religioso, entre outros);

c) distinguir a experiência turística em nível nacional e entre países, por meio de estudos comparativos;

d) estudos longitudinais que trabalhem as diversas experiências de turistas determinados ou as diferentes propostas de experiência de um empreendimento ao longo dos anos;

e) averiguar as relações entre experiência e transformação, sejam elas pessoais, de consumo, ambientais ou sociais;

f) aprofundar o entendimento dos benefícios da experiência turística.

Os estudos internacionais apresentam conclusões mais claras e, em geral, padrões de exposição e resumo dos argumentos que podem servir como boas práticas para estudos futuros. Em artigos qualitativos, há uma tendência de síntese e uso de frameworks para sintetizar os estudos. O uso de temas para descrever os resultados também é recorrente. Já em estudos quantitativos, o teste e a proposta de modelos e escalas são comuns, bem como a comparação de características demográficas da amostra.

Este trabalho não é livre de limitações. A principal delas se dá pelo recorte em seis periódicos, bem como o recorte temporal. Novas pesquisas podem abranger outras revistas e bases de dados. Ademais, apesar de se tratar de uma revisão sistemática, uma metanálise com resultados estatísticos poderia evitar possíveis vieses não intencionais. Estudos sobre os autores da área, filiações, país de origem, bem como redes sociais entre os autores, podem contribuir para a exploração dos contextos estudados. Revisões sobre os modelos teóricos já 
desenvolvidos e relações discutidas, conforme proposto por Durko e Petrick (2013), também podem servir para consolidar resultados obtidos em estudos anteriores e apresentar uma visão geral sobre os estudos da área.

O uso do Mendeley como ferramenta de apoio também demonstrou ser uma facilidade para a leitura e avaliação conjunta dos artigos. Por fim, a contribuição deste artigo não se resume à área estudada, visto que outras revisões podem utilizar processos similares aos adotados aqui para apontar lacunas para o aprimoramento das pesquisas.

\section{REFERÊNCIAS}

ALI, F.; RYU, K.; HUSSAIN, K. Influence of Experiences on Memories, Satisfaction and Behavioral Intentions: a study of creative tourism. Journal of Travel and Tourism Marketing, v. 33, n. 1, p. 85-100, 2016.

ANDERSON, L. et al. Transformative service research: an agenda for the future. Journal of Business Research, v. 66, n. 8, p. 1203-1210, 2013.

ANDRIOTIS, K. Heterotopic Erotic Oases the Public Nude Beach Experience. Annals of Tourism Research, v. 37, n. 4, p. 1076-1096, 2010.

BEHNKE, C.; SEO, S.; MILLER, K. Enhancing the study abroad experience: a longitudinal analysis of hospitalityoriented, study abroad program evaluations. Tourism Management, v. 42, p. 271-281, 2014.

BERDYCHEVSKY, L.; GIBSON, H. J. Sex and risk in young women's tourist experiences: context, likelihood, and consequences. Tourism Management, v. 51, p. 78-90, 2015.

BOJANIC, D. C. The impact of age and family life experiences on Mexican visitor shopping expenditures. Tourism Management, v. 32, n. 2, p. 406-414, 2011.

BORGES, F. R. F. Web Analytics e Gestão da Experiência do Cliente no Comércio Eletrônico: inter-relação entre os temas e utilização de um método experimental (Dissertação de Mestrado) - Universidade Federal de Minas Gerais. Departamento de Ciências Econômicas. Belo Horizonte, 2012.

BROWN, L. The Transformative Power an Ethnographic Study of the International Student Experience. Annals of Tourism Research, v. 36, n. 3, p. 502-521, 2009.

BRUNNER-SPERDIN, A.; PETERS, M. What Influences Guests' Emotions? The Case of High-quality Hotels. International Journal of Tourism Research, v. 11, p. 171-183, 2009.

BUCHMANN, A.; MOORE, K. Authenticity \& Fellowship. Annals of Tourism Research, v. 37, n. 1, p. 229-248, 2010.

BUZINDE, C. N. et al. Emic understandings of Kumbh Mela pilgrimage experiences. Annals of Tourism Research, v. 49 , p. $1-18,2014$.

CARLISLE, S. et al. Supporting innovation for tourism development through multi-stakeholder approaches: experiences from Africa. Tourism Management, v. 35, p. 59-69, 2013.

CARVALHO, D. T. et al. Experiência em website de marca-país e a formação da imagem de destino turístico: um estudo na Islândia. Experience on country brand website and the formation of tourist destination image: a study in Iceland. Experiencia en sitio web de marca país y la fo. Revista Brasileira de Pesquisa em Turismo, v. 10, n. 1, p. 108-128, 2016.

CATRAMBY, T.; BARTHOlO, R.; Delamaro, M. C. De Qué Depende El Éxito De Las Investigaciones En Turismo? Estudios y Perspectivas en Turismo, v. 22, p. 29-46, 2013.

CHANG, R. C. Y.; KIVELA, J.; MAK, A. H. N. Attributes that influence the evaluation of travel dining experience\#: when east meets west. Tourism Management, v. 32, n. 2, p. 307-316, 2011.

CHARTERS, S.; FOUNTAIN, J.; FISH, N. “You Felt Like Lingering ...”: Experiencing "Real” Service at the Winery Tasting Room. Journal of Travel Research, v. 48, n. 1, p. 122-134, 2009.

CHEN, C.-C.; HUANG, W.-J.; PETRICK, J. F. Holiday recovery experiences, tourism satisfaction and life satisfaction e Is there a relationship? Tourism Management, v. 53, p. 140-147, 2016. 
CHRONIS, A. Moving bodies and the staging of the tourist experience. Annals of Tourism Research, v. 55, p. 124$140,2015$.

COELHO, A.; RIBEIRO, L. Resenha A Economia da Experiência. Observatório de Inovação do Turismo, v. 2, n. 1, p. 1-3, 2007.

COGHLAN, A.; FILO, K. Using constant comparison method and qualitative data to understand participants' experiences at the nexus of tourism, sport and charity events. Tourism Management, v. 35, p. 122-131, 2013.

CORREIA, A.; KOZAK, M. Tourists' shopping experiences at street markets: cross-country. Tourism Management, v. 56, p. 85-95, 2016.

COUTINHO, G. C. T. P.; THOMAZ, G. M.; SAMPAIO, C. A. C. Turismo comunitário e solidário. Caderno Virtual de Turismo, v. 15, n. 1, p. 35-51, 2015.

CROUCH, G. I. et al. Discretionary Expenditure and Tourism Consumption: insights from a choice experiment. Journal of Travel Research, v. 45, n. 3, p. 247-258, 2007.

CUTLER, S. Q.; CARMICHAEL, B. The Dimensions of Customer Experience. In: MORGAN, M.; LUGOSI, P., RITCHIE, B. (Ed.). The Tourism in Leisure Experience: consumer and managerial perspectives. Bristol: Aspects of Tourism, 2010. p. 3-26.

CUTLER, S. Q.; CARMICHAEL, B.; DOHERTY, S. The Inca Trail experience: does the journey matter? Annals of Tourism Research, v. 45, p. 152-166, 2014.

DANIELS, M. J.; RODGERS, E. B. D.; WIGGINS, B. P. “'Travel Tales”: an interpretive analysis of constraints and negotiations to pleasure travel as experienced by persons with physical disabilities. Tourism Management, v. 26, n. 6, p. 919-930, 2005.

DURKO, A. M.; PETRICK, J. F. The Educational Benefits of Travel Experiences: a literature review. Journal of Travel Research, v. 52, n. 6, p. 731-744, 2013.

ECHEVERN, L. M.; ESTAY-NICULCAR, C.; ROSKER, E. Estrategias y experiencias en la construcción de marca país en américa del sur. Estudios y perspectivas en Turismo, v. 21, n. 2012, p. 288-305, 2012.

ES, N. VAN; REIJNDERS, S. Chasing sleuths and unravelling the metropolis Analyzing the tourist experience of Sherlock Holmes' London, Philip Marlowe's Los Angeles and Lisbeth Salander's Stockholm. Annals of Tourism Research, v. 57, p. 113-125, 2016.

FERDINAND, N.; WILLIAMS, N. L. International festivals as experience production systems. Tourism Management, v. 34, p. 202-210, 2013.

FERREIRA, H.; TEIXEIRA, A. A. C. "Welcome to the experience economy": assessing the influence of customer experience literature through bibliometric analysis. Working Papers (FEP) - Universidade do Porto, n. 481, p. 1-28, 2013.

GARAY, L.; CÀNOVES, G. Life Cycles, Stages and Tourism History the Catalonia (Spain ) Experience. Annals of Tourism Research, v. 38, n. 2, p. 651-671, 2011.

GOSLING, M. S.; SILVA, J. A.; COELHO, M. F. El Modelo de Experiencias aplicado a un museo la perspectiva de los visitantes. Estudios y perspectivas en turismo, v. 25, n. 4, p. 460-482, 2016.

HENNES, T. Rethinking the visitor experience: transforming obstacle into purpose. Curator: The Museum Journal, v. 45, n. 2, p. 109-121, 2002.

HERSH, M. A. Improving Deafblind Travelers' Experiences: an international survey. Journal of Travel Research, v. 55, n. 3, p. 380-394, 2016.

HOSANY, S. et al. Measuring Tourists' Emotional Experiences: further validation of the destination emotion scale. Journal of Travel Research, v. 54, n. 4, p. 482-495, 2015.

JANTA, H. et al. Employment experiences of Polish migrant workers in the UK hospitality sector. Tourism Management, v. 32, n. 5, p. 1006-1019, 2011.

KIDRON, C. A. Being There Together: dark family tourism and the emotive experience of co-presence in the holocaust past. Annals of Tourism Research, v. 41, p. 175-194, 2013. 
KIM, J.-H.; JANG, S. Memory Retrieval of Cultural Event Experiences: examining internal and external influences. Journal of Travel Research, v. 55, n. 3, p. 322-339, 2016.

KIM, J.-H.; RITCHIE, J. R. B. Cross-Cultural Validation of a Memorable Tourism Experience Scale (MTES). Journal of Travel Research, v. 53, n. 3, p. 323-335, 2014.

KOMPPULA, R.; GARTNER, W. C. Hunting as a travel experience: an auto-ethnographic study of hunting tourism in in Finland and the USA. Tourism Management, n. 35, p. 168-180, 2013.

KOMPPULA, R.; ILVES, R.; AIREY, D. Social holidays as a tourist experience in Finland. Tourism Management, v. 52, p. 521-532, 2016.

LEE, Y. Creating memorable experiences in a reuse heritage site. Annals of Tourism Research, v. 55, p. 155-170, 2015.

MACIEL, J. C. Turismo de Experiência e o sentido da vida. In: PANOSSO NETTO, A.; GAETA, C. (Ed.). Turismo de Experiência. São Paulo: Editora Senac São Paulo, 2010. p. 57-77.

MACKENZIE, S. H.; KERR, J. H. Stress and emotions at work: an adventure tourism guide's experiences. Tourism Management, v. 36, p. 2-14, 2013.

MATHIS, E. F. et al. The effect of co-creation experience on outcome variable. Annals of Tourism Research, v. 57, p. 62-75, 2016.

MELO, B. S. E o que é a Experiência de Consumo? Uma retomada na Literatura de Marketing e proposta de quadros para interesses de Futuras Pesquisas. Site Administradores.com, Trabalhos Acadêmicos, 2012.

NIEVES, S. G. La Experiencia de un Diseño Curricular en Turismo basado en un Modelo por Competencias Profesionales. Estudios y Perspectivas en Turismo, v. 19, p. 139-156, 2010.

NOVELLI, M.; SCHMITZ, B.; SPENCER, T. Networks, clusters and innovation in tourism: A UK experience. Tourism Management, v. 27, n. 6, p. 1141-1152, 2006.

OKUMUS, F.; LEVENT, A.; ROPER, A. Gaining access for research reflections from experience. Annals of Tourism Research, v. 34, n. 1, p. 7-26, 2007.

ÖZTÜRK, E. H. The role of cluster types and firm size in designing the level of network relations: the experience of the Antalya tourism region q. Tourism Management, v. 30, n. 4, p. 589-597, 2009.

PALMER, A. Customer experience management: a critical review of an emerging idea. Journal of Services Marketing, v. 24, n. 3, p. 196-208, 2010.

PALMER, C. An Ethnography of englishness experiencing identity through tourism. Annals of Tourism Research, v. 32, n. 1, p. 7-27, 2005.

PAN, T.-J. Motivations of volunteer overseas and what have we learned e the experience of Taiwanese students. Tourism Management, v. 33, n. 6, p. 1493-1501, 2012.

PANOSSO NETTO, A.; GAETA, C. (Ed.). Turismo de Experiência. São Paulo: Editora Senac São Paulo, 2010a.

PANOSSO NETTO, A.; GAETA, C. Experiência e turismo: uma união possível. In: PANOSSO NETO, A.; GAETA, C. (Ed.). Turismo de Experiência. São Paulo: Editora Senac São Paulo, 2010b. p. 43-55.

PANSIRI, J. Evolution of a doctoral thesis research topic and methodology: a personal experience. Tourism Management, v. 30, n. 1, p. 83-89, 2009.

PARK, H. YU. Shared national memory as intangible heritage: re-imagining two Koreas as one Nation. Annals of Tourism Research, v. 38, n. 2, p. 520-539, 2011.

PEZZI, E.; SANTOS, R. J. DOS. A experiência turística e o turismo de experiência: aproximações entre a antropologia e o marketing. VII SEMINÁRIO DE PESQUISA EM TURISMO DO MERCOSUL. Anais... Caxias do Sul: 2012

PEZZI, E.; VIANNA, S. L. G. A Experiência Turística e o Turismo de Experiência: um estudo sobre as dimensões da experiência memorável. Turismo em Análise, v. 26, n. 1, p. 165-187, 2015.

PINE, B. J.; GILMORE, J. H. Welcome to the Experience Economy. Harvard Business Review, July-August, p. 97 $105,1998$.

PINE, B. J. P.; GILMORE, J. H. The Experience Economy. Boston: Harvard Business Review Press, 2011. 
PORIA, Y. Assessing Gay Men and Lesbian Women's Hotel Experiences: an exploratory study of sexual orientation in the travel industry. Journal of Travel Research, v. 44, n. 3, p. 327-334, 2006.

PREBENSEN, N. K.; KIM, H. (LINA); UYSAL, M. Cocreation as Moderator between the Experience Value and Satisfaction Relationship. Journal of Travel Research, v. 55, n. 7, p. 1-12, 2016.

PRENTICE, R. C. et al. Tourism as Experience the case of Heritage Parks. Annals of Tourism Research, v. 25, $\mathrm{n}$. 1, p. 1-24, 1998.

RITCHIE, J. R. B.; TUNG, V. W. S.; RITCHIE, R. J. B. Tourism experience management research: emergence, evolution and future directions. International Journal of Contemporary Hospitality Management, v. 23, n. 4, p. 419-438, 2011.

SAMPAIO, C. A. C.; CORIOLANO, L. N. Dialogando com Experiências Vivenciadas em Marraquech e América Latina para Compreensão do Turismo Comunitário e Solidário. Revista Brasileira de Pesquisa em Turismo, v. 3, n. 1, p. 4-24, 2008.

SCARINCI, J.; PEARCE, P. The perceived influence of travel experiences on learning generic skills. Tourism Management, v. 33, n. 2, p. 380-386, 2012.

SCHMITT, B. Marketing experimental. São Paulo: Nobel, 2000.

. Gestão de Experiência do Cliente. Porto Alegre: Bookman, 2004.

SHANI, A.; URIELY, N. The Host Experience. Annals of Tourism Research, v. 39, n. 1, p. 421-440, 2012.

SHARPLEY, R.; JEPSON, D. Rural Tourism: a spiritual experience? Annals of Tourism Research, v. 38, n. 1, p. $52-$ $71,2011$.

SMALL, J.; DARCY, S.; PACKER, T. The embodied tourist experiences of people with vision impairment: management implications beyond the visual gaze. Tourism Management, v. 33, n. 4, p. 941-950, 2012.

SØRENSEN, F.; JENSEN, J. F. Value creation and knowledge development in tourism experience encounters. Tourism Management, v. 46, p. 336-346, 2015.

STONE, M. J.; PETRICK, J. F. The Educational Benefits of Travel Experiences: a literature review. Journal of Travel Research, v. 52, n. 6, p. 731-744, 2013 a.

. Health and Wellness Benefits of Travel Experiences: a literature review. Journal of Travel Research, v. 52, n. 6, p. 731-744, 2013b.

TAN, S. K.; KUNG, S. F.; LUH, D. B. A model of "creative experience" in creative tourism. Annals of Tourism Research, v. 41, p. 153-174, 2013.

TRICÁRICO, L. T. et al. Estradas-parque: um estudo comparativo no intuito de definições para a experiência turística brasileira. Revista Brasileira de Pesquisa em Turismo, v. 6, n. 1, p. 16, 2012.

TRIGO, L. G. G. A viagem como experiência significativa. In: PANOSSO NETTO, A.; GAETA, C. (Ed.). Turismo de Experiência. São Paulo: Editora Senac São Paulo, 2010.

TSENG, C. Social-demographics, driving experience and yearly driving distance in relation to a tour bus driver's atfault accident risk. Tourism Management, v. 33, n. 4, p. 910-915, 2012.

TUNG, V. W. S. et al. A framework of memory management and tourism experiences. Journal of Travel \& Tourism Marketing, v. 34, n. 7, p. 1-14, 2016.

TUNG, V. W. S.; RITCHIE, J. R. B. Investigating the Memorable Experiences of the Senior Travel Market: an examination of the reminiscence bump. Journal of Travel \& Tourism Marketing, v. 28, n. 3, p. 331-343, 2011.

TURNER, V. W. O processo ritual: estrutura e a antiestrutura. Petrópolis: Vozes, 1974.

TUSSYADIAH, I. P.; FESENMAIER, D. R. Mediating Tourist Experiences. Access to Places via Shared Videos. Annals of Tourism Research, v. 36, n. 1, p. 24-40, 2009.

VASCONCELOS, A. M. DE; LEZANA, Á. G. R. Análisis bibliométrico de la producción científica de 2002 a 2012. Estudios y Perspectivas en Turismo, v. 23, p. 645-667, 2014.

WALLS, A. R. et al. An epistemological view of consumer experiences. International Journal of Hospitality Management, v. 30, n. 1, p. 10-21, 2011. 
XUE, L.; KERSTETTER, D.; BUZINDE, C. N. Residents' experiences with tourism development and resettlement in Luoyang, China. Tourism Management, v. 46, p. 444-453, 2015.

\section{Notas}

[i]Informações disponíveis na Plataforma Sucupira, no site https://sucupira.capes.gov.br/sucupira/public/consultas/coleta/ veiculoPublicacaoQualis/listaConsultaGeralPeriodicos.jsf . Acesso em: 23/03/2017.

[ii]Conforme documento da Capes, a classificação da produção intelectual dos periódicos nacionais e internacionais varia entre A1, A2, B1, B2, B3, B4 e B5, sendo A1 o de qualidade mais elevada.

[iii]Fonte: Annals of Tourism Research. Disponível em: https://www.journals.elsevier.com/annals-of-tourism-research/. Acesso em: 27/03/2017

[iv]Fonte: Tourism Management. Disponível em: https://www.journals.elsevier.com/tourism-management . Acesso em: $27 / 03 / 2017$

[v]Fonte: Scimago Journal and Country Rank. Disponível em: http://www.scimagojr.com/journalrank.php? area $=1400 \&$ category $=1409$. Acesso em: 27/03/2017.

[vi]Os artigos retirados nesta parte da análise foram: CATRAMBY; BARTHOLO; DELAMARO, 2013; COUTINHO; THOMAZ; SAMPAIO, 2015; ECHEVERN; ESTAY-NICULCAR; ROSKER, 2012; GÓMEZ NIEVES, 2010; SAMPAIO; CORIOLANO, 2008; TRICÁRICO et al., 2012. 